\title{
Regionale Pflichtversorgung in der stationären Psychiatrie und Psychotherapie
}

\author{
Ein alternativer Ansatz zur Operationalisierung des Begriffs
}

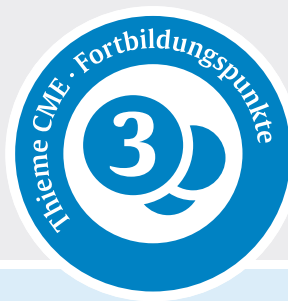

Michael Ziereis, Stefan Günther, Thomas C. Baghai, Rainer Rupprecht

Klinik für Psychiatrie und Psychotherapie, Universität Regensburg am Bezirksklinikum

\section{ZUSAMMENFASSUNG}

Gegenstand und Ziel Die vorliegende Studie zeigt einen alternativen Weg zur Operationalisierung des Begriffs „regionale Pflichtversorgung “ in der stationären Psychiatrie und Psychotherapie auf.

Material und Methoden In einer explorativen Analyse von 9659 Behandlungsfällen von psychiatrischen Fachabteilungen an 3 Standorten wurden aus Routinedaten gewonnene Kennzahlen hinsichtlich ihrer Eignung zur Abbildung des Begriffs untersucht.

Ergebnisse Im Abgleich mit einem Expertenrating konnten hohe Übereinstimmungswerte vor allem bei denjenigen Parametern gefunden werden, die den Aspekt der Vollversorgung („Diagnosespektrum“), der Notfallversorgung („Aufnahme- zeit“) und den Aspekt der Versorgungsleistung in der Region („Hauptversorgungsgebiet“) abbilden.

Schlussfolgerung Als Vorteile der Methodik werden die aufwandsneutrale Erhebungs- und Überprüfungsmechanismen sowie die vergleichsweise hohe Objektivität und Reliabilität der generierten Kennzahlen gesehen. Zudem eröffnet dieses Vorgehen die Perspektive einer Generalisierbarkeit des Begriffs der Pflichtversorgung auf andere Fachabteilungen ohne die psychiatriespezifische Besonderheit der gerichtlichen Unterbringung. Aufgrund der geringen Anzahl der betrachteten Standorte bedarf es jedoch zwingend weiterer konfirmatorischer, multizentrischer Untersuchungen.

Klinische Relevanz Ziel kann dabei die Generierung eines Komplexindex sein, welcher eine valide Abbildung des Konstrukts „regionale Pflichtversorgung“ erlaubt und damit die Basis für eine adäquate und leistungsgerechte Ressourcenzuweisung an die Kliniken vor dem Hintergrund der gesundheitspolitischen Bestrebungen für eine Neuordnung der Personalbemessung in den entsprechenden Fachgebieten schafft.

\section{Begriffsdefinition}

Laut Psychiatriebarometer 2011 [1] nehmen nach eigenen Angaben rund drei Viertel der psychiatrischen Fachkrankenhäuser und psychiatrischen Fachabteilungen an Allgemeinkrankenhäusern an der regionalen Versorgungsverpflichtung bzw. Pflichtversorgung in der stationären Psychiatrie und Psychotherapie teil. Gleichzeitig wird der Begriff der regionalen Pflichtversorgung im Rahmen der aktuellen Diskussion zur Festlegung von qualitätsorientierten Personalvorgaben immer wieder als relevanter Kostenfaktor angeführt [2, 3], da auch laut $\S 17$ d KHG „die Vereinbarung von Regelungen für Zu- oder Abschläge für die Teilnahme an der regionalen Versorgungsverpflichtung zu prüfen “ ist.

Dem steht gegenüber, dass der Begriff der regionalen Pflichtversorgung keiner abschließenden Definition oder gar Operationalisierung unterliegt. In der Psychiatrie-Enquete 1975 [4] wird erstmals der Terminus der gemeindenahen Pflichtversorgung vor dem Hintergrund von Standardversorgungsgebieten benutzt und es wird gefordert, dass geeigneten psychiatrischen stationären Krankenhauseinheiten ein Pflichtaufnahmegebiet zugewiesen werden soll. In der Regel wird der Begriff im Kontext einer gesetzlichen Verpflichtung für das Krankenhaus zur Aufnahme von psychisch kranken Menschen aus einer bestimmten Region verwendet [5, 6]. Godemann [7] nennt 2015 zusätzliche Kriterien, die zur Definition herangezogen werden könn- ten: Basierend auf der Aufnahmepflicht für Patienten, die nach den Unterbringungsgesetzen (Anm.d. V.: oder den Psychisch-Kranken-Hilfe-Gesetzen) der Länder sowie nach dem Bürgerlichen Gesetzbuch (BGB) eingewiesen werden, führt er die Merkmale der Vorhaltung von geschützten Behandlungseinheiten, der zeitnahen richterlichen Anhörung und der Überwachung durch eine Besuchskommission an. Außerdem nennt er weitere organisatorische Maßnahmen wie einen 24h-Aufnahmebetrieb oder die Vorhaltung von Kapazitätsreserven für ungeplante Aufnahmen. Schließlich wird das Vorhandensein eines durchlässigen Behandlungsangebotes aus vollstationären, teilstationären und ambulanten Angeboten als Trennkriterium vorgeschlagen. Allen Merkmalen gemeinsam ist dabei ein institutioneller und kategorialer Zugang zum Begriff der regionalen Versorgungsverpflichtung im Sinne einer klaren dichotomen Entscheidungsfindung.

\section{Patientenbezogene dimensionale Begriffsdefinition}

Vor diesem Hintergrund ist jedoch zu diskutieren, ob alle an der regionalen Pflichtversorgung teilnehmenden Krankenhäuser dies in gleicher Intensität und Breite und mit vergleichbarem finanziellem Aufwand umsetzen. Auch ist die regionale Zuständigkeit nur punktuell eindeutig geregelt [8]. Doppelzuständigkeiten oder Diskrepanzen zwi- 
- Tab. 1 Schematischer Überblick zur Operationalisierung des Begriffs der regionalen Pflichtversorgung mit Auflistung der untersuchten Parameter bzw. der daraus abgeleiteten Kennzahlen

\begin{tabular}{|c|c|c|c|c|c|}
\hline Systematik & Perspektive & Parameter & & $\S 21$ Datensatz & Kennzahl \\
\hline \multirow{11}{*}{$\begin{array}{l}\text { Operationalisierung } \\
\text { "Pflicht" }\end{array}$} & \multirow[t]{11}{*}{ Patient } & Art, Schwere, Akuität & Gerichtliche Unterbringung & nein & \\
\hline & & Art & Diagnosespektrum & ja & Kongruenz Verteilungsmuster \\
\hline & & \multirow[t]{5}{*}{ Schwere } & \multirow[t]{3}{*}{ Verweildauer } & \multirow[t]{3}{*}{ ja } & Mittelwert \\
\hline & & & & & Median \\
\hline & & & & & Standard-abweichung \\
\hline & & & \multirow{2}{*}{$\begin{array}{l}\text { Zahl der Aufnahmen } \\
\text { je Patient }\end{array}$} & \multirow[t]{2}{*}{ ja } & Mittelwert \\
\hline & & & & & Standard-abweichung \\
\hline & & \multirow[t]{4}{*}{ Akuität } & Aufnahmeanlass: Notfall & ja & Anteil \\
\hline & & & Aufnahmegrund: Notfall & ja & Anteil \\
\hline & & & \multirow[t]{2}{*}{ Aufnahmezeiten } & \multirow[t]{2}{*}{ ja } & Anteil \\
\hline & & & & & Standard-abweichung \\
\hline $\begin{array}{l}\text { Operationalisierung } \\
\text { "Regional" }\end{array}$ & Versorgung & Versorgungsregion & Hauptversorgungs-gebiet & ja & Spez. Algorithmus \\
\hline \multirow[t]{2}{*}{$\begin{array}{l}\text { Operationalisierung } \\
\text { "Versorgung“" }\end{array}$} & \multirow[t]{2}{*}{ Versorgung } & \multirow[t]{2}{*}{$\begin{array}{l}\text { Sektorenübergreifende } \\
\text { Versorgung }\end{array}$} & $\begin{array}{l}\text { Vollstationäre und } \\
\text { teilstationäre Behandlung }\end{array}$ & ja & Anteil \\
\hline & & & $\begin{array}{l}\text { Ambulante und stationäre } \\
\text { Behandlung }\end{array}$ & ja & Anteil \\
\hline
\end{tabular}

schen formeller und realer Zuständigkeit sind keinesfalls ausgeschlossen. Auch Godemann [7] erörtert in Hinblick auf strukturelle Mehrkosten, dass diese nur begrenzt von der Zuordnung einer psychiatrischen Pflichtversorgung, sondern eher von patientenbezogenen Merkmalen abhängen könnten. Deshalb wird im Folgenden eine alternative Methode zur Operationalisierung des Begriffs der regionalen Pflichtversorgung untersucht. Dieser Ansatz geht von einem dimensionalen Pflichtversorgungsbegriff aus, wodurch das einzelne Krankenhaus differenziert auf einem Kontinuum zwischen $0 \%$ und $100 \%$ Pflichtversorgung abgebildet werden kann. Die Gesamtversorgungsleistung eines Krankenhauses ergibt sich dann rechnerisch als Summation von einem elektiven Versorgungsanteil und einem Pflichtversorgungsanteil.

Die weitere Operationalisierung folgt dabei einem semantisch-inhaltlichen Ansatz. So wurden für alle Einzelaspekte des Begriffs („Pflicht“, „Regional“, „Versorgung“) mögliche Konkretisierungen aus der Perspektive der Patienten oder der Versorgung gesucht ( $\triangleright$ Tab. 1). Der Begriff „Pflicht“ wurde dabei über Parameter operationalisiert, die den Kategorien Art, Schwere oder Akuität zuzuordnen sind. Im Einzelnen waren dies das Diagnosespektrum, die Verweildauer, Wiederaufnahmerate, der Aufnahmeanlass, Aufnahmegrund und die Aufnahmezeit. Der Begriff „Regional“ wurde über einen spezifischen Algorithmus operationalisiert, der es gestattet, das Ausmaß und die Intensität der Versorgungsleistung eines Krankenhauses in einer Region zu quantifizieren. Der Begriff „Versorgung “ wurde über den Umfang der sektorenübergreifenden Versorgungsleistung erfasst. Aus der so erstellten Liste
( $\vee$ Tab. 1, Spalten 3 und 4) wurden dann diejenigen Parameter, die im Routinedatensatz gemäß § 21 KHEntgG enthalten sind, ausgewählt und über eine berechenbare Kennzahl abgebildet ( $\triangleright$ Tab. 1, Spalte 6).

Auf diese Weise konnte erreicht werden, dass die Methodik einen primär patientenbezogenen und versorgungsrelevanten Zugang zur Thematik gewährleistet und dass im Falle einer Implementierung aufgrund der ausschließlichen Nutzung von Routinedaten kein zusätzlicher Dokumentations- oder Rechtfertigungsaufwand für die Krankenhäuser entsteht.

\section{Fragestellung}

Lassen sich aus den Routinedaten der Krankenhäuser (gemäß § 21 KHEntgG) Kennzahlen aus patientenbezogenen Merkmalen identifizieren, die geeignet sind, den institutionellen Begriff der regionalen Pflichtversorgung in der Psychiatrie quantitativ abzubilden?

\section{Studienpopulation}

Eingeschlossen wurden alle im Kalenderjahr 2017 in der Versorgungsregion „Bezirk Oberpfalz“ (1,1 Mio. Einwohner) aus stationärer psychiatrischer Behandlung entlassener Patienten mit einer primären vollstationären Aufnahme in der Fachabteilung Psychiatrie und Psychotherapie/ PSY (Fallbetrachtung, $N$ = 9659). Die Population stammte dabei aus 3 Standorten unter dem Dach einer gemeinsamen Trägerstruktur. Daten zu anderen Fachabteilungen (Psychosomatik/PSO sowie Kinder- und Jugendpsychiatrie/ $\mathrm{KJP}$ ) oder zum teilstationären Behandlungssetting lagen entweder nicht für die gesamte Versorgungsregion (an- 
dere Trägerschaft) oder nur für 2 Standorte (mit eingeschränkter Beurteilbarkeit) vor.

\section{Beobachtungseinheit}

Als Beobachtungseinheit wurden alle im Kalenderjahr 2017 in den 3 Standorten aus vollstationärer psychiatrischer oder psychosomatischer Behandlung entlassenen Fälle herangezogen, deren korrespondierende Aufnahme in der Fachabteilung Psychiatrie und Psychotherapie erfolgte. Dadurch wurde ein Ausschluss derjenigen Fälle vermieden, die im Laufe ihrer Behandlung einen internen Fachabteilungswechsel von PSY nach PSO durchlaufen hatten. Da einzelne Patienten im Beobachtungszeitraum im Falle von Wiederaufnahmen auch mit mehreren Fällen in die Analyse eingegangen sind, können die Beobachtungseinheiten „Fälle“ nicht als voneinander unabhängig bewertet werden.

\section{Studientyp}

Die Untersuchung basiert auf einer beobachtenden epidemiologischen Analyse von historischen Daten im Sinne einer ökologischen Studie. Sie nutzt dazu den Routinedatensatz gemäß § 21 KHEntgG mittels sekundärer Datenanalyse.

\section{Messverfahren}

Ziel der Analyse ist es, geeignete Messinstrumente für die Fragestellung zu identifizieren, sodass die Bewertung der Validität und Güte der Methodik den Endpunkt der Untersuchung darstellt. Die erzielbare Reliabilität ist dagegen von der Art der im Detail ausgewählten Messparameter abhängig. So unterscheiden sich beispielsweise die Items Aufnahmegrund und Aufnahmezeit in diesem Punkt deutlich. Auch das Skalenniveau ist abhängig von den jeweils ausgewählten Parametern.

\section{Fallzahl}

Aufgrund des explorativen Charakters der Studie war die Fallzahl auf Basis eines Kalenderjahres limitiert ( $N=9659$ ). Eine Fallzahlsteigerung wäre zwar mittels Ausweitung des Beobachtungszeitraums möglich gewesen, jedoch nur unter Inkaufnahme einer steigenden Abhängigkeit der beobachteten Fälle. Eine weitere Fallzahlsteigerung im Rahmen einer konfirmatorischen Analyse ist mittels eines multizentrischen Studiendesigns geplant.

\section{Methode}

\section{Studienbeschreibung}

Die Studie vergleicht im Rahmen einer explorativen Analyse mögliche, kardinalskalierte Kennzahlen mit den intervallskalierten Ergebnissen eines Expertenratings [11]. Abhängig vom Übereinstimmungsgrad der beiden Bewertungsinstrumente und einer inhaltlichen Plausibilitätskontrolle wird die Eignung der Kennzahlen für die Operationalisierung des Begriffs der „regionalen Pflichtversorgung“ eingeschätzt.
- Tab.2 Charakterisierung der untersuchten Standorte: Daten aus 2017, vollstationäre Aufnahmen, Fachabteilung Psychiatrie und Psychotherapie (PSY) und Psychosomatik (PSO).

\begin{tabular}{|l|l|l|l|}
\hline & Standort A & Standort B & Standort C \\
\hline $\begin{array}{l}\text { Anzahl der Betten am } \\
\text { Standort (PSY und PSO) }\end{array}$ & 475 & 50 & 165 \\
\hline $\begin{array}{l}\text { Anzahl der Fälle mit doku- } \\
\text { mentierter gerichtlicher } \\
\text { Unterbringung }\end{array}$ & 656 & 0 & 318 \\
\hline $\begin{array}{l}\text { Somatische Klinik am } \\
\text { Standort }\end{array}$ & ja & ja & nein \\
\hline $\begin{array}{l}\text { Typisierung des Einzugs- } \\
\text { gebietes }\end{array}$ & städtisch & städtisch/ & ländlich \\
\hline
\end{tabular}

\section{Expertenrating}

- Tab. 2 zeigt die wesentlichen Charakteristika für die untersuchten Standorte A, B und C. Mangels objektiver Datengrundlage für eine graduelle Abbildung der an den 3 untersuchten Standorten realisierten Pflichtversorgungsleistung erfolgte deren Beurteilung anhand einer im Vorfeld durchgeführten Expertenschätzung mittels Rangfolgebildung auf einer intervallskalierten Skala. Demnach wurden die Standorte A, B und C wie folgt charakterisiert:

- In Anbetracht der Standortcharakteristika zu den gerichtlich untergebrachten Fällen wurde den Standorten $A$ und $C$ durch die Experten ein höherer Grad an Pflichtversorgung zugeschrieben als dem Standort B. Daraus resultiert für die untersuchten Kennzahlen K:

Prüfkriterium 1:

$\mathrm{K}(\mathrm{A})>\mathrm{K}(\mathrm{B})$ und $\mathrm{K}(\mathrm{C})>\mathrm{K}(\mathrm{B})$

Für den Fall einer negativen Korrelation der Kennzahl K wurde auch die Alternative $(K(A)<K(B)$ und $K(C)<K(B)$ untersucht.

- Unter zusätzlicher Berücksichtigung der Bettenzahl wurde der Standort C bzgl. des Umfangs der Pflichtversorgungsleistung außerdem als ähnlicher zu Standort A als zu Standort B eingestuft. Daraus resultiert für die untersuchten Kennzahlen K:

Prüfkriterium 2:

$|\mathrm{K}(\mathrm{A})-\mathrm{K}(\mathrm{C})|<|\mathrm{K}(\mathrm{A})-\mathrm{K}(\mathrm{B})|$

- Die unterschiedliche Anbindung an eine somatische Klinik und die Typisierung des Einzugsgebietes führten schließlich dazu, dass dem Standort A ein höherer Grad an Pflichtversorgung zugeschrieben wurde als dem Standort C. Daraus resultiert für die untersuchten Kennzahlen K:

Prüfkriterium 3: $\mathrm{K}(\mathrm{A})>\mathrm{K}(\mathrm{C})$ 

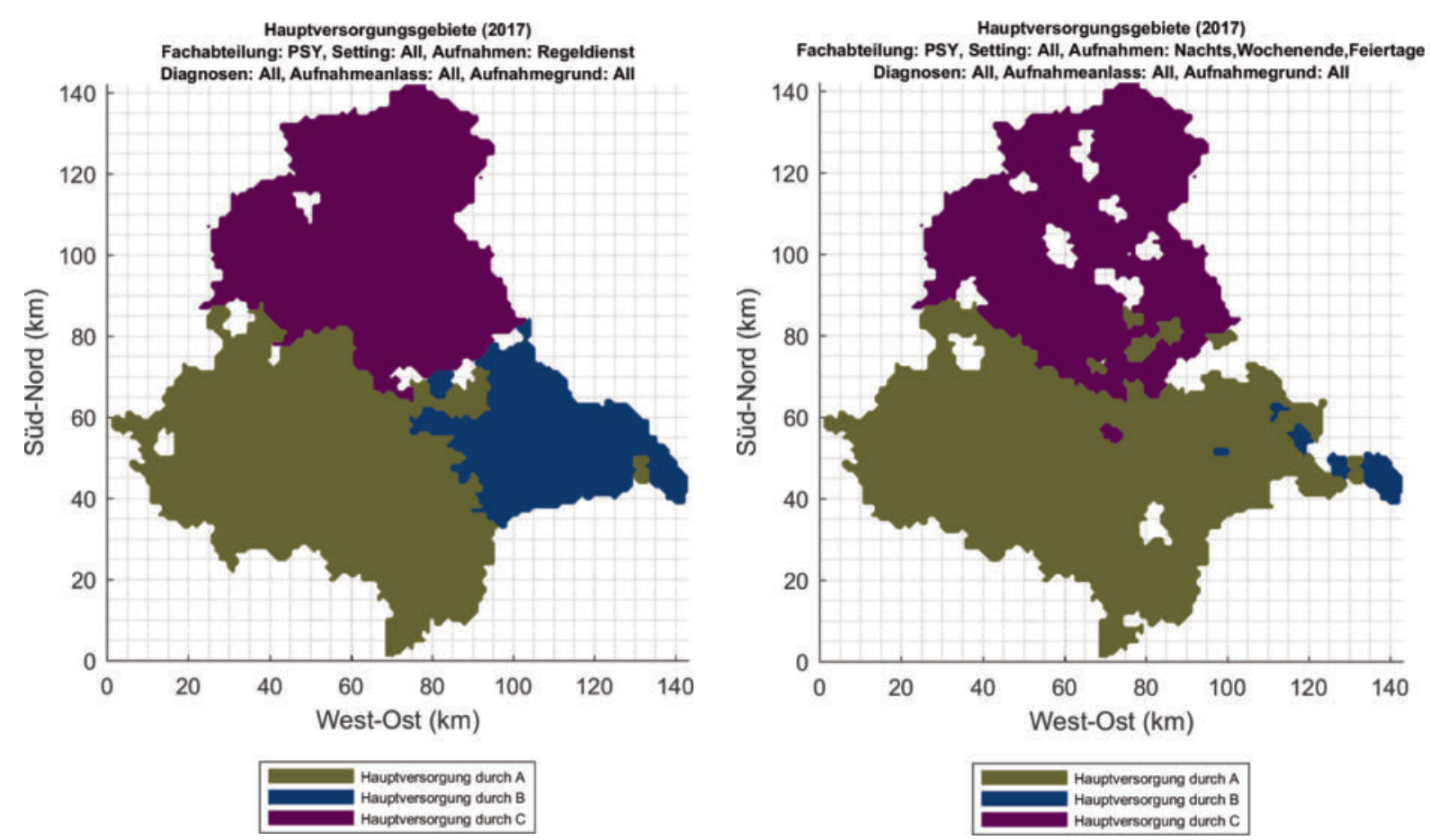

- Abb. 1 Grafische Darstellung der standortassoziierten Hauptversorgungsgebiete im Vergleich zwischen den Aufnahmen innerhalb (links) und Aufnahmen außerhalb der Kernarbeitszeit (rechts)

Auch hier wurde zusätzlich die Alternative $(K(A)<K(C)$ für den Fall einer negativen Korrelation untersucht.

\section{Primäre Kennzahlen}

Im Vorfeld war zu erwarten, dass die im Datensatz enthaltenen Parameter „Aufnahmeanlass Notfall“ und „Aufnahmegrund Notfall“ über die Abbildung der Akuität der aufgenommenen Krankheitsbilder einen Beitrag zum Grad der realisierten Pflichtversorgung liefern können. Deswegen wurde als mögliche Kennzahl der Anteil der so charakterisierten Patienten an allen am Standort aufgenommenen Patienten bestimmt ( $\triangleright$ Tab. 1).

Analog wurde auch der Anteil aller außerhalb der regulären Dienstzeiten (hier 7:00 Uhr bis 19:00 Uhr) aufgenommenen Patienten sowie die Streuung der Aufnahmezeiten (Stundenwerte) untersucht. Laut Hamburg Center for Health Economics [9, 10] kann der Parameter „Anzahl Fälle außerhalb der Kernarbeitszeit (vollstationär)“ als Indikator für Notfälle genutzt werden.

Als möglicherweise mit dem Schweregrad der Erkrankung assoziierte Parameter wurden außerdem die Lagemaße (Mittelwert bzw. Median) und Streuungsmaße zur Verweildauer und zur Zahl der Aufnahmen je Patient bestimmt. Um die Art der aufgenommenen Krankheitsbilder mittels einer einzigen Kennzahl abbilden zu können, wurde das auf Basis der 2-stelligen ICD-10-Hauptdiagnose normierte Di- agnoseverteilungsmuster der Standorte mit dem analog normierten Verteilungsmuster der InEK-Kalkulationsstichprobe für den Fachabteilung Psychiatrie und Psychotherapie [12] abgeglichen und daraus ein Übereinstimmungsgrad $(0=$ keine Übereinstimmung bis $1,0=$ vollständige Übereinstimmung mit der InEK-Stichprobe) errechnet.

Für die Abbildung der sektorenübergreifenden Versorgungsleistung wurde zum einen der Anteil an Patienten bestimmt, die im Beobachtungszeitraum sowohl vollstationär als auch teilstationär in den Standorten behandelt worden sind, zum anderen wurde der Anteil an Patienten bestimmt, die sowohl stationär als auch ambulant behandelt worden sind.

Zur Operationalisierung des Regionalitätsaspektes wurde ein spezifischer Rechenalgorithmus angewandt, der in einem ersten Schritt je Postleitzahl denjenigen Standort ermittelte, der die meisten Patienten mit dieser Postleitzahl als Wohnsitz aufnahm (Hauptversorger). In einem zweiten Schritt wurde dann je Standort der Anteil derjenigen Patienten bestimmt, die aus den zugehörigen Hauptversorgungsgebieten aufgenommen worden sind. - Abb. 1 zeigt links die Visualisierung der Hauptversorgungsgebiete der untersuchten Standorte. Nicht zugeordnete Areale („weiße Flächen“) resultieren dabei aus einer numerischen Äquivalenz der aufgenommenen Patienten aus verschiedenen Standorten. 
> Tab. 3 Übersicht zu den errechneten Kennzahlen für die untersuchten Standorte (A, B und C) für alle Aufnahmen sowie differenziert nach Aufnahmen innerhalb und außerhalb der regulären Dienstzeit.

\begin{tabular}{|c|c|c|c|c|c|c|c|c|c|c|}
\hline & & \multicolumn{3}{|c|}{ Alle Aufnnahmen } & \multicolumn{3}{|c|}{$\begin{array}{l}\text { Aufnahmen innerhalb der } \\
\text { regulären Dienstzeit }\end{array}$} & \multicolumn{3}{|c|}{$\begin{array}{l}\text { Aufnahmen außerhalb der } \\
\text { regulären Dienstzeit }\end{array}$} \\
\hline & & $\begin{array}{l}\text { Stand- } \\
\text { ort A }\end{array}$ & $\begin{array}{l}\text { Stand- } \\
\text { ort B }\end{array}$ & $\begin{array}{l}\text { Stand- } \\
\text { ort C }\end{array}$ & $\begin{array}{l}\text { Stand- } \\
\text { ort A }\end{array}$ & $\begin{array}{l}\text { Stand- } \\
\text { ort B }\end{array}$ & $\begin{array}{l}\text { Stand- } \\
\text { ort C }\end{array}$ & $\begin{array}{l}\text { Stand- } \\
\text { ort A }\end{array}$ & $\begin{array}{l}\text { Stand- } \\
\text { ort B }\end{array}$ & $\begin{array}{l}\text { Stand- } \\
\text { ort C }\end{array}$ \\
\hline $\begin{array}{l}\text { Diagnose- } \\
\text { spektrum }\end{array}$ & $\begin{array}{l}\text { Kongruenz } \\
\text { Verteilungs- } \\
\text { muster }\end{array}$ & 0,89 & 0,57 & 0,82 & 0,89 & 0,56 & 0,81 & 0,83 & 0,61 & 0,77 \\
\hline \multirow{3}{*}{$\begin{array}{l}\text { Verweildauer } \\
\text { (in Tagen) }\end{array}$} & Mittelwert & 28,62 & 33,85 & 24,97 & 32,02 & 33,84 & 26,47 & 23,85 & 35,28 & 20,55 \\
\hline & Median & 19,00 & 35,00 & 19,00 & 22,00 & 35,00 & 21,00 & 11,00 & 34,00 & 13,00 \\
\hline & $\begin{array}{l}\text { Standard- } \\
\text { abweichung }\end{array}$ & 36,17 & 17,96 & 26,71 & 37,92 & 16,57 & 26,60 & 33,35 & 29,91 & 24,95 \\
\hline \multirow{2}{*}{$\begin{array}{l}\text { Zahl der Auf- } \\
\text { nahmen } \\
\text { je Patient }\end{array}$} & Mittelwert & 1,35 & 1,31 & 1,35 & 1,43 & 1,31 & 1,40 & 1,53 & 1,60 & 1,51 \\
\hline & $\begin{array}{l}\text { Standard- } \\
\text { abweichung }\end{array}$ & 0,80 & 0,63 & 0,74 & 0,88 & 0,61 & 0,79 & 0,99 & 1,06 & 0,92 \\
\hline $\begin{array}{l}\text { Aufnahme- } \\
\text { anlass: Notfall }\end{array}$ & Anteil & 0,64 & 0,70 & 0,29 & 0,36 & 0,65 & 0,10 & 0,27 & 0,05 & 0,19 \\
\hline $\begin{array}{l}\text { Aufnahme- } \\
\text { grund: Notfall }\end{array}$ & Anteil & 0,64 & 0,70 & 0,27 & 0,35 & 0,65 & 0,07 & 0,29 & 0,06 & 0,20 \\
\hline \multirow[t]{2}{*}{$\begin{array}{l}\text { Aufnahme- } \\
\text { zeiten }\end{array}$} & Anteil & $\begin{array}{l}\text { keine } \\
\text { Aus- } \\
\text { sage- } \\
\text { kraft }\end{array}$ & $\begin{array}{l}\text { keine } \\
\text { Aus- } \\
\text { sage- } \\
\text { kraft }\end{array}$ & $\begin{array}{l}\text { keine } \\
\text { Aus- } \\
\text { sage- } \\
\text { kraft }\end{array}$ & 0,64 & 0,92 & 0,72 & 0,36 & 0,08 & 0,28 \\
\hline & $\begin{array}{l}\text { Standard- } \\
\text { abweichung }\end{array}$ & 5,03 & 3,14 & 4,61 & 2,58 & 2,09 & 2,57 & 7,33 & 8,28 & 7,06 \\
\hline $\begin{array}{l}\text { Hauptversor- } \\
\text { gungsgebiet }\end{array}$ & $\begin{array}{l}\text { Spez. } \\
\text { Algorithmus }\end{array}$ & 0,93 & 0,54 & 0,88 & 0,91 & 0,77 & 0,87 & 0,96 & 0,27 & 0,92 \\
\hline $\begin{array}{l}\text { Vollstationäre } \\
\text { und teil- } \\
\text { stationäre } \\
\text { Behandlung }\end{array}$ & Anteil & 0,04 & 0,07 & 0,05 & 0,05 & 0,07 & 0,05 & 0,00 & 0,00 & 0,00 \\
\hline $\begin{array}{l}\text { Ambulante } \\
\text { und stationäre } \\
\text { Behandlung }\end{array}$ & Anteil & 0,33 & 0,76 & 0,24 & 0,37 & 0,76 & 0,27 & 0,29 & 0,70 & 0,19 \\
\hline
\end{tabular}

\section{Sekundäre (bedingte) Kennzahlen}

Aus der Liste aller möglichen bedingten Kennzahlen (z. B. Verweildauer unter der Bedingung einer Aufnahme aus dem Hauptversorgungsgebiet) wurden für alle Kennzahlen auch die Bedingungen „Aufnahmen innerhalb der regulären Dienstzeit“, und „Aufnahmen außerhalb der regulären Dienstzeit geprüft. Als Aufnahme außerhalb der regulären Dienstzeit galt dabei jede Aufnahme zwischen 19:00 Uhr und 7:00 Uhr sowie an Wochenenden und an Feiertagen. \ Abb. 1 zeigt im Seitenvergleich beispielhaft den Einfluss der genannten Bedingungen auf die Berechnung des Hauptversorgungsgebietes.

\section{Bewertung}

Alle primären und sekundären (bedingten) Kennzahlen wurden abschließend hinsichtlich der Erfüllung der Prüf- kriterien 1 bis 3 aus dem Expertenrating untersucht, sodass daraus eine Bewertung zwischen 0 und 3 Punkten je Kennzahl resultierte.

\section{Ergebnisse}

- Tab. 3 zeigt die ermittelten Kennzahlen für die 3 Standorte im Überblick. Exemplarisch ist der Tabelle zu entnehmen, dass der Übereinstimmungsgrad des Diagnosespektrums für alle Aufnahmen mit der InEK-Stichprobe am Standort A bei $89 \%$, am Standort B bei $57 \%$ und am Standort C bei 82 \% liegt. Während die Kennzahlen für die Aufnahmen innerhalb der regulären Dienstzeit nahezu identische Werte liefern, finden sich bei den Aufnahmen außerhalb der regulären Dienstzeit je nach Standort ge- 


\begin{tabular}{|c|c|c|c|c|}
\hline & & Alle Aufnnahmen & $\begin{array}{l}\text { Aufnahmen innerhalb der } \\
\text { regulären Dienstzeit }\end{array}$ & $\begin{array}{l}\text { Aufnahmen außerhalb der } \\
\text { regulären Dienstzeit }\end{array}$ \\
\hline Diagnosespektrum & $\begin{array}{l}\text { Kongruenz } \\
\text { Verteilungsmuster }\end{array}$ & 3 & 3 & 3 \\
\hline \multirow[t]{3}{*}{ Verweildauer } & Mittelwert & 2 & 2 & 2 \\
\hline & Median & 2 & 2 & 3 \\
\hline & Standardabweichung & 2 & 2 & 0 \\
\hline \multirow{2}{*}{$\begin{array}{l}\text { Zahl der Aufnahmen je } \\
\text { Patient }\end{array}$} & Mittelwert & 3 & 3 & 2 \\
\hline & Standardabweichung & 3 & 3 & 2 \\
\hline Aufnahmeanlass: Notfall & Anteil & 2 & 2 & 3 \\
\hline Aufnahmegrund: Notfall & Anteil & 2 & 2 & 3 \\
\hline \multirow[t]{2}{*}{ Aufnahmezeiten } & Anteil & keine Aussagekraft & 3 & 3 \\
\hline & Standardabweichung & 3 & 3 & 2 \\
\hline Hauptversorgungsgebiet & Spez. Algorithmus & 3 & 3 & 3 \\
\hline $\begin{array}{l}\text { Vollstationäre und teil- } \\
\text { stationäre Behandlung }\end{array}$ & Anteil & 3 & 3 & 0 \\
\hline $\begin{array}{l}\text { Ambulante und stationäre } \\
\text { Behandlung }\end{array}$ & Anteil & 2 & 2 & 2 \\
\hline
\end{tabular}

- Abb. 2 Bewertung der untersuchten Kennzahlen unter Anwendung der Prüfkriterien des Expertenratings. Eine Bewertung mit 0 (rot) bedeutet dabei, dass keines der drei Prüfkriterien erfüllt ist, eine Bewertung mit 3 (grün) zeigt dagegen an, dass alle 3 Prüfkriterien erfüllt sind.

ringe Abweichungen von obigen Werten mit jeweils unterschiedlichem Vorzeichen.

In > Abb. 2 finden sich die Bewertungsergebnisse für die Indikatoren. Im Beispiel erfüllen demnach die Standortkennzahlen für die Kongruenz des Diagnosespektrums alle Prüfkriterien 1 bis 3 - unabhängig davon, ob alle Aufnahmen oder nur die Aufnahmen innerhalb oder nur die Aufnahmen außerhalb der regulären Dienstzeit betrachtet werden. Anhand der Farbkodierung ist unschwer zu erkennen, dass die geringste Übereinstimmung zwischen Kennzahl und Expertenrating bei den Parametern „Mittelwert der Verweildauer“ und „Sektorenübergreifende Versorgung: Ambulante und stationäre Behandlung“ erzielt werden konnte.

Die Kennzahl „Sektorenübergreifende Versorgung: Vollstationäre und teilstationäre Behandlung “ zeigt eine deutlich bessere Übereinstimmung - allerdings mit negativer Korrelation. So korrespondieren unter 2 der 3 gewählten Bedingungen hohe Werte für die sektorenübergreifende Versorgung mit einem niedrigen Expertenrating zur Pflichtversorgung.

Die Kennzahlen „Aufnahmeanlass Notfall“ und „Aufnahmegrund Notfall“ erfüllten nur bei Betrachtung der Aufnahmen außerhalb der regulären Dienstzeit alle Bedingungen des Expertenratings. Bei der Nachbetrachtung zeigten sich außerdem standortspezifisch unterschiedliche Gepflogenheiten bei der Zuweisung dieser Kodierungen im Rahmen des administrativen Aufnahmeprozesses.
Bei den verweildauerbasierten Kennzahlen „Median Verweildauer" und „Streuung Verweildauer" fanden sich unter 2 von 3 Bedingungen jeweils 2 von 3 Prüfkriterien erfüllt. Tendenziell waren dabei in den Standorten mit einer höheren Zuschreibung an Pflichtversorgung Patienten, die außerhalb der regulären Dienstzeit aufgenommen wurden, kürzer in Behandlung als Patienten, die innerhalb der regulären Dienstzeit aufgenommen wurden. Eine komplette Übereinstimmung der Kennzahlen mit dem Expertenrating fand sich bei 2 von 3 Bedingungen bei den Parametern „Mittelwert Zahl der Aufnahmen je Patient“, „Streuung Zahl der Aufnahmen je Patient“, „Anteil Aufnahmezeiten“ und „Streuung Aufnahmezeiten“.

Schließlich konnte bei den Kennzahlen „Diagnosespektrum: Kongruenz Verteilungsmuster“ und „Hauptversorgungsgebiet“ unter allen 3 Aufnahmebedingungen eine vollständige Übereinstimmung zwischen den ermittelten Werten und dem Expertenrating gefunden werden.

\section{Diskussion}

Die Studie zeigt einen alternativen Weg zur Operationalisierung des Begriffs „regionale Pflichtversorgung“ auf. Verwendung finden dabei nur Routinedaten auf Individualebene, die mittels einfacher Algorithmen zu Kennzahlen für den jeweiligen Krankenhausstandort transformiert werden. Hierdurch entsteht kein zusätzlicher Erhebungsaufwand für die Klinken und kein zusätzlicher Überprüfungsaufwand für die Kostenträger und den MDK. 
Die objektive Aussagekraft dieser Daten übersteigt dabei die Validität der per Selbstauskunft zur Teilnahme an der regionalen Pflichtversorgung vorliegenden Informationen deutlich. So kann etwa mit dem Algorithmus des Hauptversorgungsgebietes das tatsächliche Versorgungsgebiet - unabhängig von dem politisch zugewiesenen Gebiet - dargestellt und quantifiziert werden. Auch können bestehende regionale Unterschiede (z. B. zwischen Stadt und Land) durch Berechnung der Kennzahlen unter der Bedingung der jeweiligen Hauptversorgungsregion besser berücksichtigt werden.

In der untersuchten Stichprobe fand sich eine unbefriedigende Abbildung des Pflichtversorgungsbegriffs durch die Fallklassifikation „Notfall“. Ursache dafür können fehlende Ausfüll- und Kodierhinweise sein. Derzeit ist daher nicht von einer ausreichenden Durchführungsobjektivität und Reliabilität für diesen Parameter auszugehen.

Auch fand sich keine positive Korrelation zwischen dem Umfang der am jeweiligen Standort erbrachten sektorenübergreifenden Versorgung und der Experteneinschätzung zum Umfang der erbrachten Pflichtversorgungsleistung. Insbesondere die Eignung der Kennzahl zur sektorenübergreifenden voll- und teilstationären Behandlung muss bei Vorliegen einer negativen Korrelation aus inhaltlicher Sicht kritisch gesehen werden.

Auch die Verwendung der Zahl der Aufnahmen je Patient als Kennzahl ist trotz des Vorliegens einer hohen Übereinstimmung zwischen dem ermittelten Wert und dem Expertenrating versorgungspolitisch zu hinterfragen. Es wäre zu prüfen, ob mittels diagnosespezifischer Stratifizierung noch ein Beitrag zur Operationalisierung des Parameters geleistet werden kann. Darüber hinaus fanden sich hohe Übereinstimmungswerte zwischen den ermittelten Kennzahlen und dem Expertenrating vor allem bei den Parametern, die den Aspekt der Vollversorgung („Diagnosespektrum: Kongruenz Verteilungsmuster“) der Notfallversorgung („Aufnahmezeiten“) und den Aspekt der Versorgungsleistung in der Region („Hauptversorgungsgebiet“) abbilden.

In diesem Zusammenhang ist zudem anzumerken, dass eine stärkere Ausrichtung des Pflichtversorgungsbegriffs auf diese Aspekte anstelle der Fokussierung auf die Zahl der gerichtlichen Unterbringungen sogar positiv bewertet werden kann. Neben den methodischen Vorteilen eröffnet dieses Vorgehen die Perspektive einer Generalisierbarkeit des Begriffs der Pflichtversorgung auf andere Fachabteilungen ohne die psychiatriespezifische Besonderheit der gerichtlichen Unterbringung.

Allen Indikatoren (Ausnahme: Kennzeichnung als „Notfall“) kann dabei eine ausreichende Objektivität und Reliabilität zugeschrieben werden („Diagnosespektrum“ nur eingeschränkt). Die inhaltliche Plausibilität und Validität der Indikatoren ist dadurch gewährleistet, dass die Definition des zu prüfenden Konstrukts erst eingangs - unter Abgleich mit den späteren Prüfkriterien - erarbeitet worden ist. Die Gültigkeit der vorgeschlagenen Indikatoren für davon abweichende Begriffsdefinitionen zur regionalen Pflichtversorgung ist nicht beurteilbar.

Zur weiteren Evaluation der vorgeschlagenen Kennzahlen bedarf es als nächsten Schritt der Ausweitung der Untersuchung auf eine größere Anzahl von Standorten und Trägern. Im Rahmen eines multizentrischen Designs ist sowohl eine konfirmatorische statistische Analyse als auch der Einbezug der Fachabteilungen der Psychosomatik und der Kinder- und Jugendpsychiatrie in die Analyse sicherzustellen. Anstelle eines Expertenratings sollte das Vergleichsranking dann auf Basis der Ergebnisse einer Kostenträgerrechnung erfolgen, sodass auch der graduell-dimensionale Charakter der Kennzahlen evaluiert werden kann.

Neben der endgültigen Auswahl von geeigneten Kennzahlen verbleibt abschließend noch die Aufgabe, diese mittels Gewichtung oder gegenseitige Bedingtheit zu einem Komplexindex zusammenzuführen, welcher eine valide Abbildung des Konstrukts „regionale Pflichtversorgung“ erlaubt und dann auch Basis für eine adäquate Ressourcenzuweisung sein kann.

Beispielhaft können auf Basis der in der Studie imponierenden Kennzahlen „Diagnosespektrum: Kongruenz Verteilungsmuster (außerhalb der regulären Arbeitszeit) “ und „Hauptversorgungsgebiet (außerhalb der regulären Arbeitszeit)“ unter der Prämisse der gleichen Gewichtung und Adjustierung der Kennzahlen folgende Ergebnisse für die untersuchten Standorte errechnet werden:

Standort A: $=0,90$
Standort B: $=0,44$
Standort C: $=0,85$

Die so ermittelten Werte geben ein sehr gutes Abbild des Expertenratings zu den Standorten wieder und erlauben somit eine valide Abbildung des Konstrukts „regionale Pflichtversorgung“ gemäß der hier vorgeschlagenen Definition.

Angesichts der derzeitigen gesundheitspolitischen Weichenstellungen, die zu einer Neuordnung der Personalbemessung in der Fächern Psychiatrie und Psychotherapie, Psychosomatik und Kinder- und Jugendpsychiatrie führen sollen, ist eine objektivierbare Operationalisierung des Begriffs „Pflichtversorgung“ von höchster Relevanz. 


\section{FAZIT FÜR DIE PRAXIS}

- Der Begriff der „regionalen Pflichtversorgung“ ist grundsätzlich dimensional abbildbar.

- Eine Operationalisierung ist unter alleinigem Rückgriff auf Routinedaten möglich.

- Als relevant erweisen sich die Aspekte der Vollversorgung, der Notfallversorgung und der Versorgungsleistung in der Region.

- Die Methodik kann die Basis für eine leistungsgerechte Ressourcenzuweisung liefern.

- Weitere konfirmatorische Studien sind notwendig.

\section{Interessenkonflikt}

Erklärung zu finanziellen Interessen

Forschungsförderung erhalten: ja; Honorar/geldwerten Vorteil für Referententätigkeit erhalten: ja; Bezahlter Berater/interner Schulungsreferent/Gehaltsempfänger: ja; Patent/Geschäftsanteile/Aktien (Autor/Partner, Ehepartner, Kinder) an Firma (Nicht-Sponsor der Veranstaltung): nein; Patent/Geschäftsanteile/Aktien (Autor/Partner, Ehepartner, Kinder) an Firma (Sponsor der Veranstaltung): nein.

Erklärung zu nicht finanziellen Interessen

Die Autoren erklären, dass keine Interessenkonflikte im Zusammenhang mit dieser Veröffentlichung bestehen.

\section{Korrespondenzadresse}

\section{Dr. med. Michael Ziereis}

Klinik für Psychiatrie und Psychotherapie der Universität Regensburg am Bezirksklinikum Universitätsstraße 84, 93053 Regensburg Tel. 0941/9411622

Michael.Ziereis@medbo.de

\section{Literatur}

[1] Blum K, Löffert S, Offermanns M et al. Psychiatrie Barometer Umfrage 2011. Im Internet: https://www.dki.de/sites/default/files/2019-02/psychbarometer_2011.pdf

[2] Bundesministerium der Justiz und für Verbraucherschutz, Verordnung über Maßstäbe und Grundsätze für den Personalbedarf in der stationären Psychiatrie (Psychiatrie-Personalverordnung - Psych-PV), 1990. Im Internet: https://www. gesetze-im-internet.de/psych-pv/BJNR029300990.html
[3] Hauth I, Brückner-Bozetti P, Heuft G et al. Personalausstattung in stationären psychiatrischen Einrichtungen. Nervenarzt 2019; 90: 285-292. doi.org/10.1007/s00115018-0669-z

[4] Deutscher Bundestag. Zur psychiatrischen und psychotherapeutischen/psychosomatischen Versorgung der Bevölkerung. Drucksache 1975, 7/4200. Im Internet: http://dipbt. bundestag.de/doc/btd/07/042/0704200.pdf

[5] Gemeinsamer Bundesausschuss. Glossar 2016. Im Internet: https://www.g-ba.de/downloads/17-98-4124/2016-06-16_ Erlaeuterung-Qb-R_Glossar.pdf

[6] Arbeitsgruppe Psychiatrie der Obersten Landesgesundheitsbehörden 2007, Psychiatrie in Deutschland - Strukturen, Leistungen, Perspektiven, Gesundheitsministerkonferenz der Länder (Hrsg.). Im Internet: https://www.gmkonline. de/_beschluesse/Protokoll_80-GMK_Top1002_Anlage1_Psychiatrie-Bericht.pdf

[7] Godeman F, Hauth I, Richert A et al. Merkmale einer regionalen Pflichtversorgung in Deutschland. Nervenarzt 2015; 86: 367. doi.org/10.1007/s00115-015-4257-1

[8] Ministerium für Arbeit, Gesundheit und Soziales des Landes Nordrhein-Westfalen, Datenbank zu den Pflichtversorgungsgebieten in der Kinder- und Jugendpsychiatrie. Im Internet: https://www.mags.nrw/datenbank-pflichtversorgungsgebiete-psychiatrie

[9] Hamburg Center for Health Economics (HCHE) in Zusammenarbeit mit BQS Institut für Qualität \& PatientensicherheitBegleitforschung zu den Auswirkungen der Einführung des pauschalierenden Entgeltsystems für psychiatrische und psychosomatische Einrichtungen, Endbericht für die Datenjahre 2011-2012. Im Internet: https://www.g-drg.de/ Datenbrowser_und_Begleitforschung/Begleitforschung_Psychiatrie/Endbericht_Nullpunktmessung

[10] Hamburg Center for Health Economics (HCHE) in Zusammenarbeit mit BQS Institut für Qualität \& Patientensicherheit, Begleitforschung zu den Auswirkungen der Einführung des pauschalierenden Entgeltsystems für psychiatrische und psychosomatische Einrichtungen, Endbericht für die Datenjahre 2011-2015. Im Internet: https://www.g-drg.de/ Datenbrowser_und_Begleitforschung/Begleitforschung_ Psychiatrie/Endbericht_1._Forschungszyklus_Datenjahre_2013_2015

[11] Vandenbroucke JP, von Elm E, Altman DG et al. Strengthening the Reporting of Observational Studies in Epidemiology (STROBE): Explanation and Elaboration. Epidemiology 2007; 18 (6): 805-35. PMID: 18049195

[12] InEK GmbH - Institut für das Entgeltsystem im Krankenhaus 2010-2017. Im Internet: https://www.g-drg.de/Datenbrowser_und_Begleitforschung/PEPP-Browser/PEPP-Browser_2018

Bibliografie

DOI https://doi.org/10.1055/a-0952-7171

Nervenheilkunde 2020; 39: 167-174

(c) Georg Thieme Verlag KG Stuttgart · New York ISSN 0722-1541 


\section{Punkte sammeln auf CM/F.thieme.de}

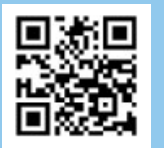

Diese Fortbildungseinheit ist bis zu 12 Monate online für die Teilnahme verfügbar.

Den genauen Einsendeschluss finden Sie beim Modul auf https://cme.thieme.de/CXDEF]1.

Sollten Sie Fragen zur Online-Teilnahme haben, finden Sie unter https://cme.thieme.de/hilfe

eine ausführliche Anleitung. Wir wünschen viel Erfolg beim Beantworten

der Fragen!

Unter https://eref.thieme.de/CXDEFJ1 oder über den QR-Code kommen Sie

direkt zum Artikel.

VNR 2760512020158722344

\section{Frage 1}

Wie viele psychiatrische Fachkrankenhäuser und Fachabteilungen nehmen nach eigenen Angaben an der regionalen Versorgungsverpflichtung in der stationären Psychiatrie und Psychotherapie teil?
A Ca. $25 \%$
B Ca. $50 \%$
C Ca. $75 \%$
D Ca. $90 \%$
E Ca. $95 \%$

\section{Frage 2}

Welche Aussage trifft zu?

A Der Begriff der regionalen Pflichtversorgung wurde im Rahmen der Psychiatrie-Enquete für obsolet erklärt.

B Der Begriff der regionalen Pflichtversorgung ist derzeit klar definiert.

C Der Begriff der regionalen Pflichtversorgung ist derzeit gut operationalisiert.

D Der Begriff der regionalen Pflichtversorgung spielt bei der Bemessung von Zu- und Abschlägen laut §17 d KHG keine Rolle.

E Der Begriff der regionalen Pflichtversorgung wird derzeit kategorial im Sinne einer ja/nein Entscheidung für eine Institution verwendet.

\section{Frage 3}

Welche Aussage trifft nicht zu? Als mögliche Kriterien für das Vorliegen einer regionalen Versorgungsverpflichtung werden in der Literatur genannt:

A Aufnahmepflicht für Patienten, die nach den Unterbringungsgesetzen der Länder eingewiesen sind.

B Aufnahmepflicht für Patienten, die nach dem Bürgerlichen Gesetzbuch eingewiesen sind.

C Aufnahmepflicht für Patienten, die nach dem Maßregelvollzugsgesetz eingewiesen sind.

D Vorhaltung von Kapazitätsreserven für ungeplante Aufnahmen.

E Vorhaltung eines 24-h-Aufnahmebetriebes.

\section{Frage 4}

Welche Aussage trifft nicht zu? Die Routinedaten des § 21 KHEntG enthalten unter anderem folgende Angaben:

A Die mögliche Kennzeichnung eines Patienten als „Notfall“.

B Angaben zum Aufnahmezeitpunkt des Patienten.

C Die mögliche Kennzeichnung eines Patienten als „gerichtlich untergebracht“.

D Angaben zur Diagnose eines Patienten.

E Angaben zur Verweildauer eines Patienten.

\section{Frage 5}

Welche Aussage trifft zu? Das standortassoziierte Versorgungsgebiet eines Krankenhauses

A ist grundsätzlich nicht darstellbar.

$B$ ist immer eindeutig einem einzelnen Krankenhaus zuzuordnen.

C kann sich in Abhängigkeit vom Beobachtungszeitraum unterschiedlich darstellen.

D kann nur durch das Krankenhaus selbst dargestellt werden.

E weist stets eine Übereinstimmung zwischen formeller und realer Zuständigkeit aus.

\section{Frage 6}

Welche Aussage trifft zu? Die Zahl der stationären Aufnahmen je Patient

A kommt als mögliches Maß zur Beurteilung der Schwere der Erkrankung eines Patienten in Betracht.

B ist ausschließlich von der Schwere der Erkrankung eines Patienten abhängig.

$C$ ist ausschließlich von der Diagnose eines Patienten abhängig.

D zeigt keine Korrelation zur Pflichtversorgungsleistung eines Krankenhauses.

E ist ein geeignetes Maß zur Beurteilung der Pflichtversorgungsleistung eines Krankenhauses. 


\section{Punkte sammeln auf CME.thieme.de}

Fortsetzung ...

\section{Frage 7}

Welche Aussage trifft nicht zu? Laut vorliegender Studie erweisen sich als geeignet für die Operationalisierung des Begriffs der "regionalen Pflichtversorgung":

A Der Aspekt der Vollversorgung durch das Krankenhaus.

B Der Aspekt der Notfallversorgung durch das Krankenhaus (Aufnahme von Patienten in der Nacht).

C Der Aspekt der Notfallversorgung durch das Krankenhaus (Aufnahme von Patienten am Wochenende).

D Der Aspekt der Versorgungsleistung des Krankenhauses in der Region.

E Der Aspekt der Vorhaltung einer sektorenübergreifenden Versorgungskette (stationär und teilstationär) durch das Krankenhaus.

\section{Frage 8}

Welche Aussage trifft zu? Die Operationalisierung des Begriffs der „Regionale Pflichtversorgung“

A kann grundsätzlich nicht dimensional (im Sinne einer graduellen Entscheidung) erfolgen.

B ist unter alleinigem Rückgriff auf Routinedaten möglich.

C geht zwangsläufig mit einem zusätzlichen Dokumentationsaufwand für die Kliniken einher.

D geht zwangsläufig mit einem höheren Überprüfungsaufwand durch die Kostenträgern einher.

E geht zwangsläufig mit einem höheren Überprüfungsaufwand durch den MDK einher.

\section{Frage 9}

Welche Aussage trifft nicht zu? Eine valide Operationalisierung der regionalen Pflichtversorgungsleistung eines Krankenhauses

A kann perspektivisch die Basis für eine leistungsgerechte Ressourcenzuweisung liefern.

B kann perspektivisch nicht ohne die Berücksichtigung der gerichtlichen Unterbringung gelingen.

C kann perspektivisch auch auf anderer Fachabteilungen wie z.B. die Kinder- und Jugendpsychiatrie generalisiert werden.

D kann perspektivisch auch auf anderer Fachabteilungen wie z.B. die Psychosomatik generalisiert werden.

E kann perspektivisch mittels eines Komplexindex je Standort erfolgen.

\section{Frage 10}

Welche Aussage trifft nicht zu? Die Ergebnisse der vorliegenden Studie

A bedürfen einer konfirmatorischen Ausweitung auf eine größere Anzahl an Krankenhausstandorten.

$B$ bedürfen einer konfirmatorischen Ausweitung auf eine größere Anzahl an Krankenhausträgern.

C bedürfen einer Ausweitung des Vergleichsrankings auf Basis der Ergebnisse einer Kostenträgerrechnung.

D bedürfen einer konfirmatorischen Ausweitung unter Einbezug von multiplen Expertenratings.

E bedürfen einer konfirmatorischen Ausweitung der statistischen Analyse. 